\title{
A Note on the Effect of Negative Poisson's Ratio on the Deformation of a Poroelastic Half-Space by Surface Loads
}

\author{
Sunita Rani ${ }^{1}$, Raman Kumar ${ }^{1}$, Sarva Jit Singh ${ }^{2}$ \\ ${ }^{1}$ Department of Mathematics, Guru Jambheshwar University of Science and Technology, Hisar, India \\ ${ }_{2}^{2}$ Honorary Scientist, Indian National Science Academy, New Delhi, India \\ E-mail:s_b_rani@rediffmail.com, $s_{\_} j_{-}$singh@yahoo.com \\ Received January 8, 2010; revised March 4, 2010; accepted March 9, 2010
}

\begin{abstract}
The aim of this note is to study the effect of negative Poisson's ratio on the quasi-static deformation of a poroelastic half-space with anisotropic permeability and compressible fluid and solid constituents by surface loads. Two particular cases considered are: two-dimensional normal strip loading and axisymmetric normal disc loading. It is found that a negative Poisson's ratio makes the Mandel-Cryer effect more prominent. It also results in an increase in the magnitude of the surface settlement.
\end{abstract}

Keywords: Anisotropic Permeability, Auxetic Material, Negative Poisson's Ratio, Poroelastic Half-Space, Quasi-Static Deformation, Surface Loading

\section{Introduction}

A homogeneous, isotropic, poroelastic medium can be characterized by four constitutive parameters. Let these parameters be: the shear modulus $(G)$, the drained Poisson's ratio $(v)$, the undrained Poisson's ratio $\left(v_{u}\right)$ and the Biot-Willis coefficient $(\alpha)$, with $-1 \leq v \leq v_{u} \leq 0.5$. For highly compressible pore fluid $v=v_{u}$ and for incompressible fluid and solid constituents $v_{u}=0.5$. Materials with negative Poisson's ratio were unknown until Lakes [1] showed that such materials can be artificially created. In a series of papers, Lakes and his coworkers have investigated interesting properties of materials with negative Poisson's ratio. Negative Poisson's ratio materials are also called auxetic materials or auxetics [2]. Yang et al. [3] presented a review on auxetic materials.

The dynamic behaviour of auxetic materials has been studied by many investigators. The effect of negative Poisson's ratio on several dynamic problems of elasticity was studied by Lipsett \& Beltzer [4]. Chen \& Lakes [5] examined possible use of auxetic materials for viscoelastic damping applications. Freedman [6] studied the effect of negative Poisson's ratio on Lamb modes in a free plate. Chen \& Lakes [7] compared the dynamic wave dispersion and loss properties of conventional and auxetic polymeric cellular materials. Scarpa et al. [8] claim an overall superiority regarding damping and acoustic properties of auxetic polyurethane foams as compared to the original conventional foam. They suggest possible use of auxetic foams in the field of antivibration pads and gloves. Auxetic materials also offer very good sound and vibration absorption and could have many applications in aerospace and defence areas.

Ting \& Barnett [9] derived simple necessary and sufficient conditions on elastic compliances to identify if any given anisotropic material of cubic or hexagonal symmetry is completely auxetic or nonauxetic. Stability of an elastic material with negative stiffness and negative Poisson's ratio has been discussed by Shang \& Lakes [10]. In a recent paper, Kocer et al. [11] investigated the properties of a layered composite, with alternating layers of materials with negative and positive Poisson's ratio. They observed an increase in the resistance to mechanical deformation above the average value of the two constituent materials. Therefore, it might be possible to fabricate materials, with a designed microstructure, using both conventional and auxetic materials, for specific engineering applications. Possible applications of auxetic materials are based on their superior toughness, shear resistance, indentation resistance and fracture toughness.

Motivation for the present study was provided by $\mathrm{Ku}-$ rashige et al. [12]. Foams with negative Poisson's ratio are being considered as possible future advanced materials for a variety of applications. Further, poroelastic materials have interesting properties due to coupling between elastic skeletel deformation and pore fluid diffusion. It is, therefore, useful to study the response of foam to sudden loading if it possesses a negative Poisson's 
ratio and is saturated with a fluid.

Kurashige et al. [12] studied four Mandel and Cryer problems for fluid-saturated auxetic foams, assuming the displacement field as irrotational. Sawaguchi and Kurashige [13] studied the transient response of a poroelastic circular cylinder subjected to axial compression with a constant strain rate. They showed that if the Poisson's ratio of the cylinder is negative, the surface of the cylinder extends in the tangential direction immediately after loading but stops extending after a while. Thereafter, the surface of the cylinder experiences tangential contraction.

The purpose of the present note is to study numerically the effect of negative Poisson's ratio on the diffusion of the pore pressure and time-settlement of a poroelastic half-space with anisotropic permeability and compressible fluid and solid constituents by two-dimensional strip loading or axisymmetric disc loading. For this purpose, we use the analytical expressions for the pore pressure and the vertical displacement derived recently by Singh et al. $[14,15]$ and calculate these quantities by numerical integration.

\section{Theory}

\subsection{Normal Strip Loading}

Consider a strip $-L \leq x \leq L$ of infinite length in the $y$-direction on the surface of a permeable poroelastic half-space $z \geq 0$. Let a normal load $\sigma_{0}$ per unit length acting in the positive z-direction be uniformly distributed over the strip. Then the Laplace transform of the pore fluid pressure at any point of the half-space is given by Singh et al. [14]

$$
\begin{aligned}
& \bar{p}= \\
& \frac{\left(v_{u}-v\right) s \sigma_{0}}{\pi \eta} \int_{0}^{\infty}\left(e^{-m z}-e^{-k z}\right) \frac{k+m}{\Omega} \cos k x\left(\frac{\sin k L}{k L}\right) d k
\end{aligned}
$$

where

$s=$ Laplace transform variable

$$
\begin{gathered}
\eta=\frac{1-2 v}{2(1-v)} \alpha \\
m=\left(\frac{c_{1}}{c_{3}} k^{2}+\frac{s}{c_{3}}\right)^{1 / 2}
\end{gathered}
$$

$c_{1}=$ hydraulic diffusivity in the horizontal direction

$c_{3}=$ hydraulic diffusivity in the vertical direction

$$
\begin{gathered}
\Omega=s\left(v_{u}-v\right)(k-m)-s_{a}\left(1-v_{u}\right)(k+m) \\
s_{a}=s+\left(c_{1}-c_{3}\right) k^{2} .
\end{gathered}
$$

We are assuming the half-space to be poroelastic with anisotropic permeability and compressible fluid and solid constituents. In the particular case of isotropic permeability, $c_{1}=c_{3}$. For incompressible fluid and solid constituents, $\alpha=1, v_{u}=0.5$.

On taking the limit $s \rightarrow \infty$, we get the pore pressure for the undrained state (fast loading, short-term behaviour). In this case, the integral appearing in (1) can be evaluated analytically, yielding

$$
p_{u}=\frac{\left(v_{u}-v\right) \sigma_{0}}{\alpha(1-2 v) \pi L} \tan ^{-1}\left(\frac{2 L z}{z^{2}+x^{2}-L^{2}}\right) .
$$

In (3) it is assumed that $z>0, x^{2}+z^{2} \geq L$. However, if $x^{2}+z^{2}<L^{2}$, we must add $\pi$ to $\tan ^{-1}$ ( ) term appearing in (3).

\subsection{Normal Disc Loading}

Suppose a total normal load $Q_{0}$ is uniformly applied in the positive $z$-direction over a circular surface area $(z=0$, $r \leq a$ ) of a radius with its centre at the origin. If the surface $(z=0)$ of the half-space is permeable, the Laplace transform of the pore fluid pressure given by Singh et al. [15] is

$$
\begin{aligned}
& \bar{p}= \\
& \frac{\left(v_{u}-v\right) s Q_{0}}{\pi a \eta} \int_{0}^{\infty}\left(e^{-m z}-e^{-k z}\right) J_{0}(k r) J_{1}(k a)\left(\frac{m+k}{\Omega}\right) d k .
\end{aligned}
$$

The vertical (down) displacement in the Laplace transform domain is

$$
\begin{aligned}
& \bar{w}= \\
& \frac{Q_{0}}{\pi a G} \int_{0}^{\infty}\left[\frac{\left(v_{u}-v\right) m s}{k-m}\left(e^{-m z}-e^{-k z}\right)-\frac{1}{2}\left\{s\left(v_{u}-v\right)+s_{a}\left(1-v_{u}\right)\right\}\right. \\
& \left.\times(m+k) z e^{-k z}-(1-v)\left(1-v_{u}\right) s_{a}\left(\frac{m+k}{k}\right) e^{-k z}\right] \\
& \times J_{0}(k r) J_{1}(k a) \frac{1}{\Omega} d k
\end{aligned}
$$

On taking the limit $s \rightarrow \infty$ and evaluating the integrals we get the pore pressure and the vertical displacement for the undrained state in the form

$$
\begin{gathered}
p_{u}= \\
\frac{2\left(v_{u}-v\right) Q_{0}}{\pi a^{2} \eta(1-v)} \sum_{n=0}^{\infty}(-1)^{n}\left(\begin{array}{c}
2 \mathrm{n}+1 \\
\mathrm{n}
\end{array}\right)\left[\frac{a^{2}}{4\left(r^{2}+z^{2}\right)}\right]^{n+1} \\
\times P_{2 n+1}\left(\frac{z}{\sqrt{z^{2}+r^{2}}}\right), \\
w_{u}=\frac{Q_{0}}{\pi a G}\left[\frac{z}{a} \sum_{n=0}^{\infty}(-1)^{n}\left(\begin{array}{c}
2 \mathrm{n}+1 \\
\mathrm{n}
\end{array}\right)\left\{\frac{a^{2}}{4\left(r^{2}+z^{2}\right)}\right\}^{n+1}\right.
\end{gathered}
$$




$$
\begin{gathered}
\times P_{2 n+1}\left(\frac{z}{\sqrt{z^{2}+r^{2}}}\right)+\left(1-v_{u}\right) \\
\left.\times \sum_{n=0}^{\infty} \frac{(-1)^{n} 2 n !}{n !(n+1) !}\left\{\frac{a^{2}}{4\left(r^{2}+z^{2}\right)}\right\}^{n+\frac{1}{2}} P_{2 n}\left(\frac{z}{\sqrt{z^{2}+r^{2}}}\right)\right],
\end{gathered}
$$

where $P_{n}(x)$ is the Legendre polynomial of degree $n$ and

$$
\left(\begin{array}{l}
n \\
r
\end{array}\right)=\frac{n !}{r !(n-r) !} .
$$

\section{Numerical Results}

The expressions for the pore pressure and the vertical displacement given by (1), (4) and (5) are in the transform domain. Two integrations are required to be performed to get the corresponding expressions in the physical domain. We have used Schapery's approximate formula [16] for the Laplace inversion and the extended Simpson's rule for evaluating the infinite integrals over $k$.

\subsection{Normal Strip Loading}

We introduce the following dimensionless quantities:

$$
\begin{gathered}
P=\left(\frac{L}{\sigma_{0}}\right) p, \quad Z=\frac{z}{L}, \\
T=\left(\frac{G \chi_{3}}{L^{2}}\right) t, \quad \gamma=\left(\frac{c_{1}}{c_{3}}\right)^{1 / 2} .
\end{gathered}
$$

Figures $1(\mathbf{a}, \mathbf{b}, \mathbf{c})$ show the effect of the Poisson ratio $(v)$ on the diffusion of the pore pressure with time at the point $x=0, z=2 L$ on the $z$-axis, assuming $v_{u}=0.31, \alpha=$ 0.65 and $\gamma=0.1,1,10$. Figure 1 (b) is for isotropic permeability $\left(\gamma=1, c_{1}=c_{3}\right)$. From (3), the undrained pore pressure is independent of the anisotropy parameter $\gamma$ and for $x=0, z=2 L$, has the value

$$
P_{u}=\frac{v_{u}-v}{\pi \alpha(1-2 v)} \tan ^{-1}\left(\frac{4}{3}\right) .
$$

This is a decreasing function of $v$ for $-1 \leq v \leq v_{u} \leq 0.5$. The undrained pore pressure for $v=-0.25$ is more than three times its value for $v=0.25$.

In Figures $1(\mathbf{a}, \mathbf{b}, \mathbf{c})$, we have plotted $P$ as a function of time for five value of $v$. We notice that, for a given $T$, the pore pressure increases as $v$ decreases. For all $v$, the pore pressure, instead of decreasing monotonically with time, rises above the initial undrained value before it decays to zero as $T \rightarrow \infty$. This is in accordance with the Mandel-Cryer effect [17]. This effect is more pronounced for smaller values of the Poisson's ratio ( $v)$. In

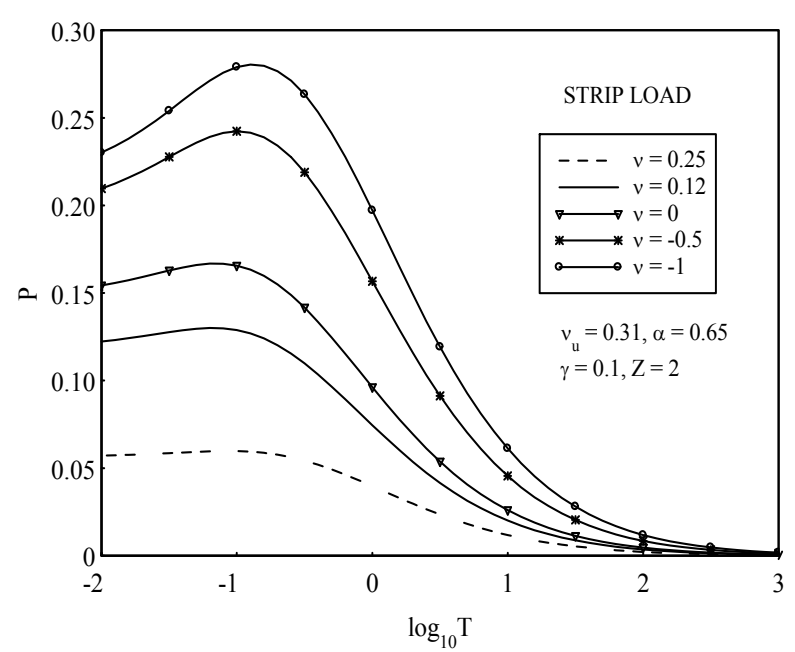

(a)

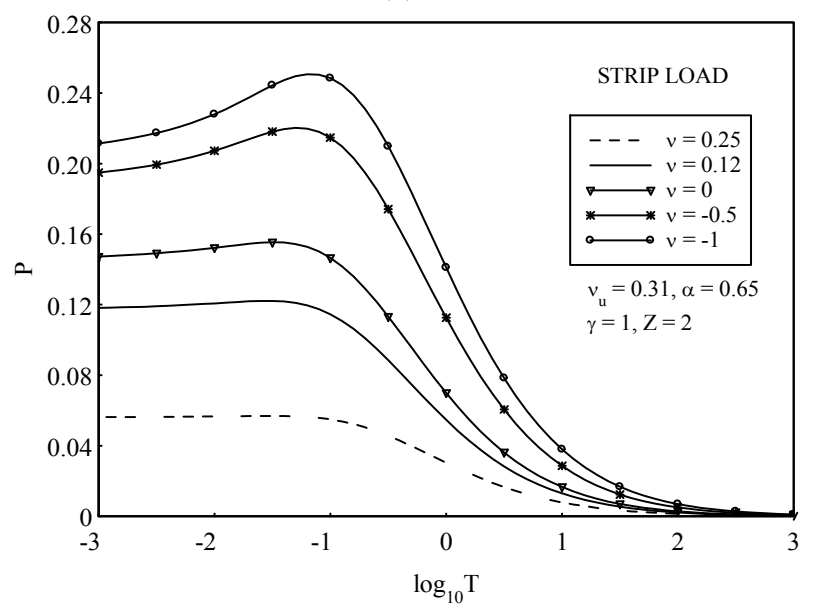

(b)

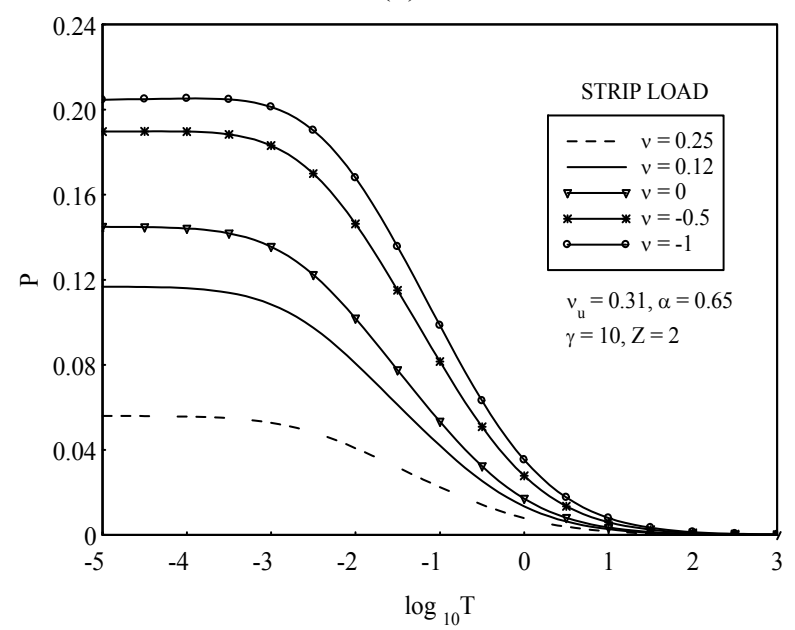

(c)

Figure 1. Effect of the Poisson's ratio ( $v$ ) on the diffusion of the pore pressure $(P)$ with time $(T)$ for $v_{u}=0.31, \alpha=0.65, z$ $=2 a$ and (a) $\gamma=0.1$, (b) $\gamma=1$, (c) $\gamma=10$ for normal strip loading. 
particular, the Mandel-Cryer effect is much more prominent for a poroelastic half-space with a negative Poisson's ratio $(v)$ than for a poroelastic half-space with a positive $v$.

Figures $2(\mathbf{a}, \mathbf{b}, \mathbf{c})$ show the depth profile of the pore

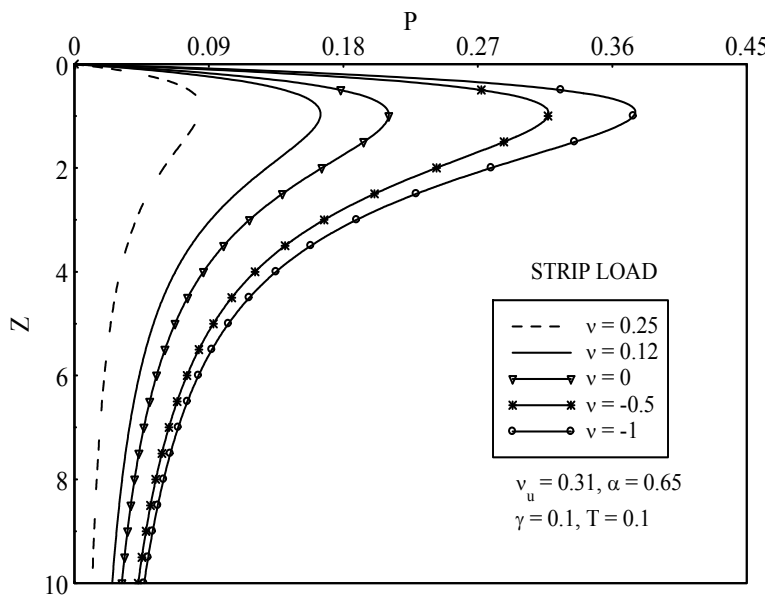

(a)

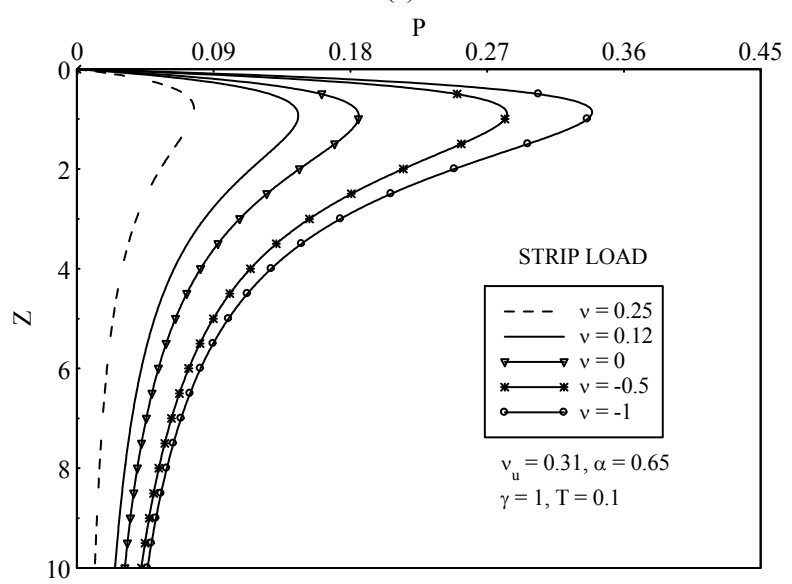

(b)

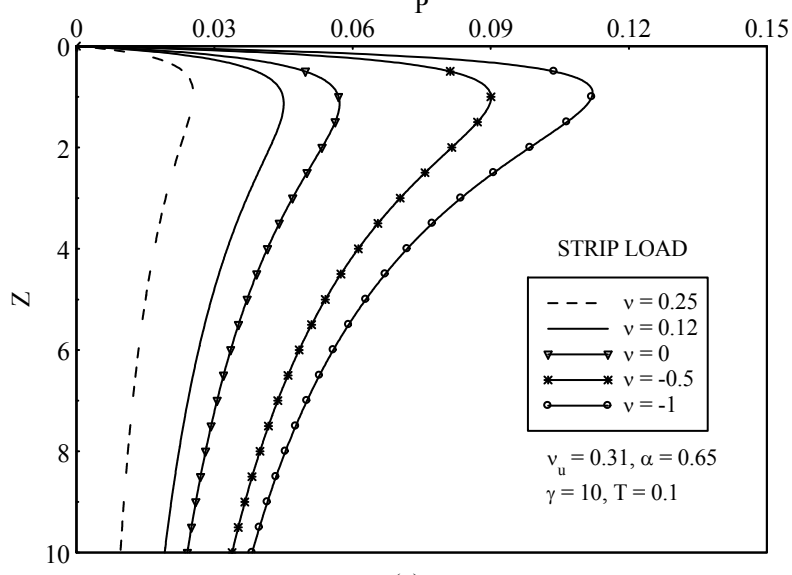

(c)

Figure 2. Effect of the Poisson's ratio on the depth profile of the pore pressure at $T=0.1$ for $v_{u}=0.31, \alpha=0.65$ and (a) $\gamma$ $=0.1$, (b) $\gamma=1$, (c) $\gamma=10$ for normal strip loading. pressure for $v_{u}=0.31, \alpha=0.65, T=0.1$ and $\gamma=0.1,1$, 10 for five values of $v$. The pore pressure is zero at the surface, attains a maximum at a depth depending upon $\gamma$ and $T$ and then tends to zero as $Z \rightarrow \infty$. Here again, we notice that, at all depths, the pore pressure increases as $v$ decreases.

\subsection{Normal Disc Loading}

We introduce the following dimensionless quantities:

$$
\begin{gathered}
P=\left(\frac{\pi a^{2}}{Q_{0}}\right) p, \quad W=\left(\frac{\pi a G}{Q_{0}}\right) w, \quad Z=\frac{z}{a} \\
T=\left(\frac{\chi_{3} G}{a^{2}}\right) t, \quad \gamma=\left(\frac{c_{1}}{c_{3}}\right)^{1 / 2} .
\end{gathered}
$$

Figures $3(\mathbf{a}, \mathbf{b}, \mathbf{c})$ show the time-settlement at the centre of the disc load $(r=0, z=0)$ for $v_{u}=0.31, \alpha=$ $0.65, \gamma=0.1,1,10$ for five values of $v=-1,-0.5,0,0.12$, 0.25 . For $t>0$, as $v$ decreases, the surface settlement increases. In particular, the surface settlement for a halfspace with a negative Poisson's ratio $(v)$ is much more than for a half-space with a positive $v$. The undrained surface settlement is independent of the Poisson's ratio v.

Figures 4 (a, b, c) display the influence of the Poisson's ratio $(v)$ on the diffusion of the pore pressure with time at the point $r=0, z=2 a$ on the axis of symmetry for three values of the anisotropy parameter $\gamma=0.1,1,10$. From (6), the undrained pore pressure at the point $r=0, z$ $=2 a$ is

$$
\begin{gathered}
P_{u}=\frac{\left(v_{u}-v\right)}{\alpha(1-2 v)} \sum_{n=0}^{\infty}\left[(-1)^{n}\left(\begin{array}{c}
2 n+1 \\
n
\end{array}\right)\left(\frac{1}{4}\right)^{2 n+1}\right] \\
=\frac{\left(v_{u}-v\right)(2-4 / \sqrt{5})}{\alpha(1-2 v)}
\end{gathered}
$$

which is a decreasing function of $v$ for $-1 \leq v \leq v_{u} \leq 0.5$. The pore pressure vanishes in the drained state (slow loading, long-term behaviour). As in the case of normal strip loading, we notice that the pore pressure increases as the Poisson's ratio decreases. The effect of a decrease in $v$ is to enhance the Mandel-Cryer effect. In particular, in a poroelastic half-space with a negative Poisson's ratio $(v)$, this effect is much more prominent than for a poroelastic half-space with a positive $v$.

\section{Conclusions}

For almost all common materials the Poisson's ratio is positive. However, in 1987, R. S. Lakes showed that materials possessing negative Poisson's ratio can be artificially created. We have studied the effect of negative 


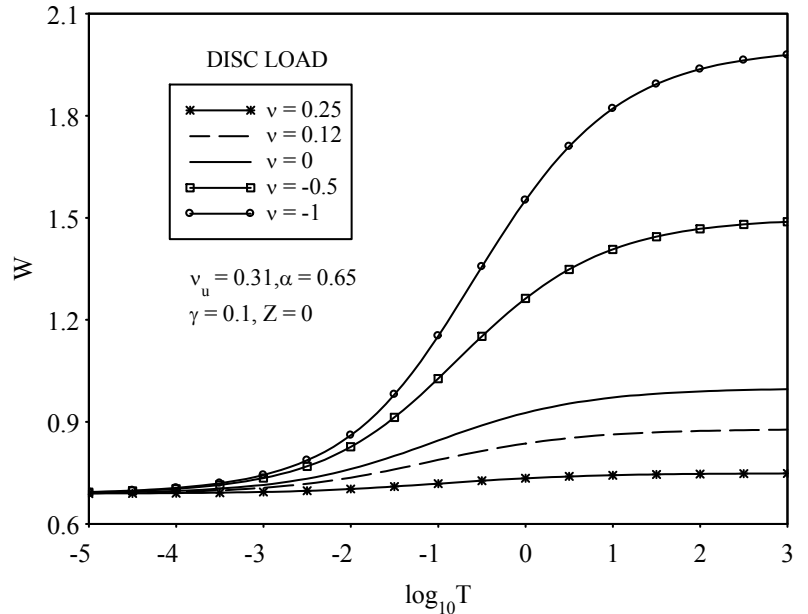

(a)

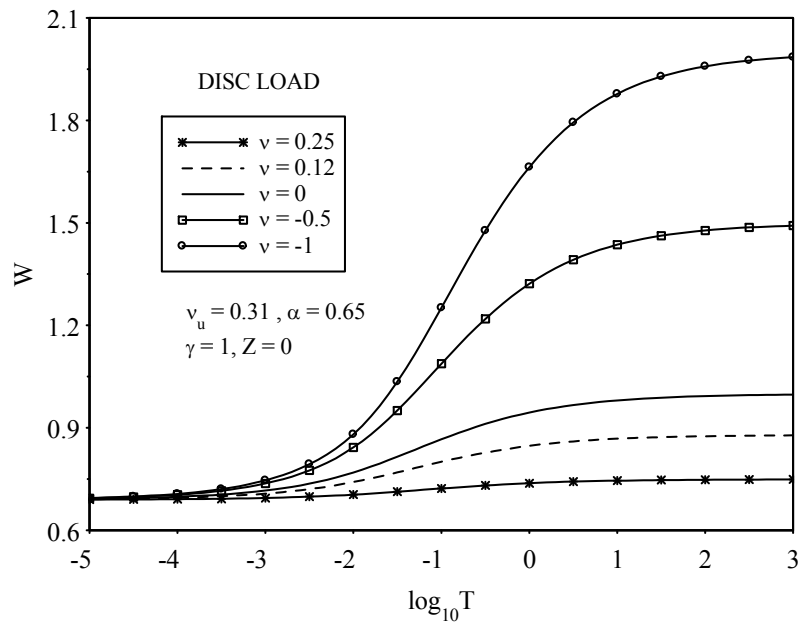

(b)

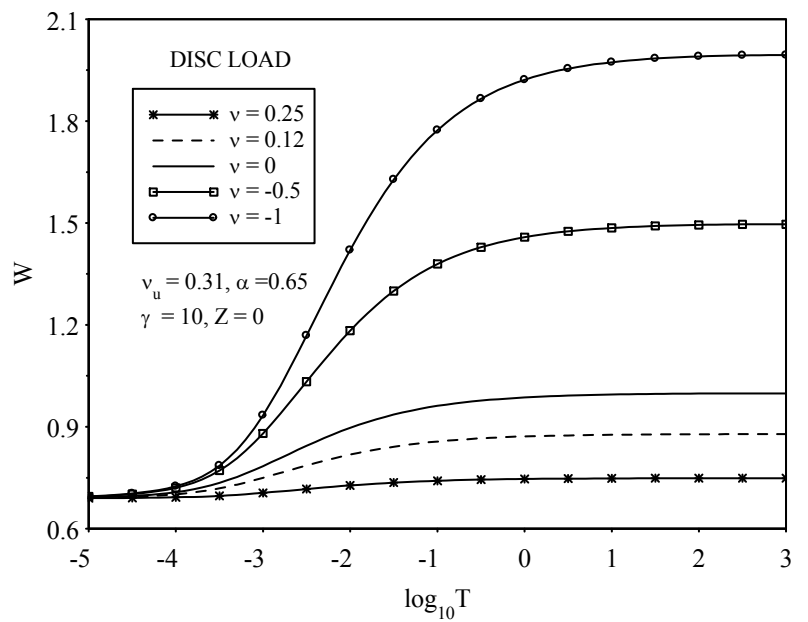

(c)

Figure 3. Effect of the Poisson's ratio (v) on the time-settlement for $v_{u}=0.31, \alpha=0.65, z=0$ and (a) $\gamma=0.1$, (b) $\gamma=1$, (c) $\gamma=10$ for normal disc loading.

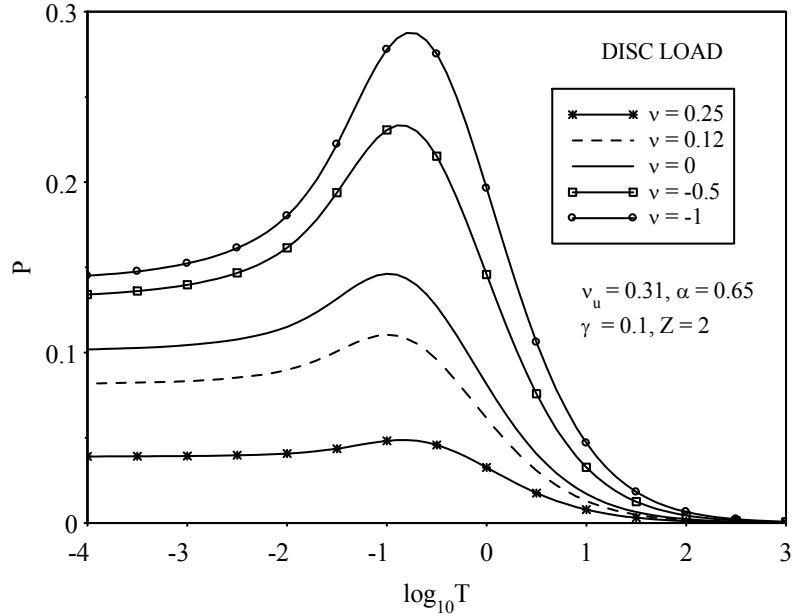

(a)

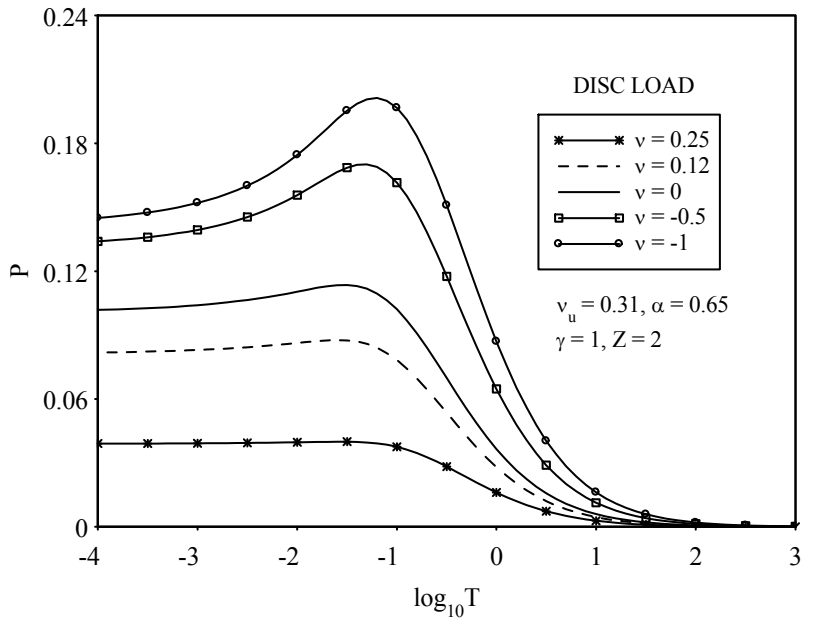

(b)

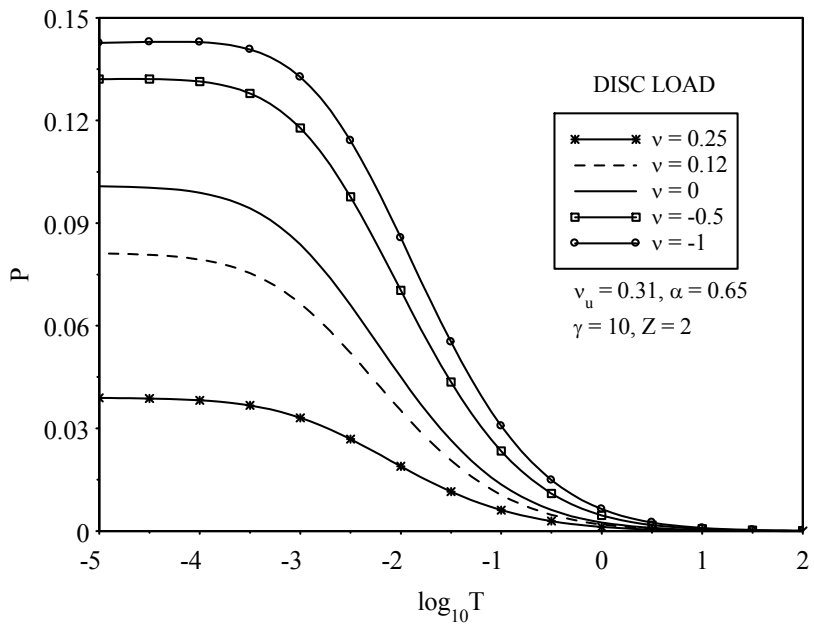

(c)

Figure 4. Effect of the Poisson's ratio $(v)$ on the diffusion of the pore pressure $(P)$ with time $(T)$ for $v_{u}=0.31, \alpha=0.65, z$ $=2 a$ and (a) $\gamma=0.1$, (b) $\gamma=1$, (c) $\gamma=10$ for normal disc loading. 
Poisson's ratio on the consolidation of a poroelastic half-space by the surface loads. It has been observed that in case of negative Poisson's ratio, Mandel-Cryer effect is more pronounced. It also results in an increase in the magnitude of the surface settlement.

\section{Acknowledgements}

SJS thanks the Indian National Science Academy for financial support.

\section{References}

[1] R. S. Lakes, "Foam Structures with a Negative Poisson's Ratio," Science, Vol. 235, No. 27, 1987, pp. 1038-1040.

[2] B. D. Caddock and K. E. Evans, "Microporous Materials with Negative Poisson's Ratios: I. Microstructure and Mechanical Properties," Journal of Physics D: Applied Physics, Vol. 22, No. 12, 1989, pp. 1877-1882.

[3] W. Yang, Z.-M. Li, W. Shi, B.-H. Xie and M.-B. Yang, "Review on Auxetic Materials," Journal of Materials Science, Vol. 39, No. 10, 2004, pp. 3269-3279.

[4] A. W. Lipsett and A. I. Beltzer, "Reexamination of Dynamic Problems of Elasticity for Negative Poisson's Ratio," The Journal of the Acoustical Society of America, Vol. 84, No. 6, 1988, pp. 2179-2186.

[5] C. P. Chen and R. S. Lakes, "Dynamic Wave Dispersion and Loss Properties of Conventional and Negative Poisson's Ratio Polymeric Cellular Materials," Cellular Polymers, Vol. 8, No. 5, 1989, pp. 343-359.

[6] A. Freedman, "The Variation, with the Poisson's Ratio, of Lamb Modes in a Free Plate, I, General Spectra," Journal of Sound and Vibration, Vol. 137, No. 2, 1990, pp. 209-230.

[7] C. P. Chen and R. S. Lakes, "Micromechanical Analysis of Dynamic Behavior of Conventional and Negative Poisson's Ratio Foams," Journal of Engineering Materials and Technology, Vol. 118, 1996, pp. 285-288.

[8] F. Scarpa, L. G. Ciffo and J. R. Yates, "Dynamic Properties of High Structural Integrity Auxetic Open Cell
Foam," Smart Materials and Structures, Vol. 13, No. 1, 2004, pp. 49-56.

[9] T. C. T. Ting and D. M. Barnett, "Negative Poisson's Ratios in Anisotropic Linear Elastic Media," Journal of Applied Mechanics, Vol. 72, No. 6, 2005, pp. 929-931.

[10] X. Shang and R. S. Lakes, "Stability of Elastic Material with Negative Stiffness and Negative Poisson's Ratio," Physica Status Solidi B, Vol. 244, No. 3, 2007, pp. 1008-1026.

[11] C. Kocer, D. R. McKenzie and M. M. Bilek, "Elastic Properties of a Material Composed of Alternating Layers of Negative and Positive Poisson's Ratio," Materials Science and Engineering: A, Vol. 505, No. 1-2, 2009, pp. 111-115.

[12] M. Kurashige, M. Sato and K. Imai, "Mandel and Cryer Problems of Fluid-Saturated Foams with Negative Poisson's Ratio," Acta Mechanica, Vol. 175, No. 1-4, 2005, pp. 25-43.

[13] H. Sawaguchi and M. Kurashige, "Constant Strain-Rate Compression Test of a Fluid-Saturated Poroelastic Sample with Positive or Negative Poisson's Ratio," Acta Mechanica, Vol. 179, No. 3-4, 2005, pp. 145-156.

[14] S. J. Singh, S. Rani and R. Kumar, "Quasi-Static Deformation of a Poroelastic Half-Space with Anisotropic Permeability by Two-Dimensional Surface Loads," Geophysical Journal International, Vol. 170, No. 3, 2007, pp. 1311-1327.

[15] S. J. Singh, R. Kumar and S. Rani, "Consolidation of a Poroelastic Half-Space with Anisotropic Permeability and Compressible Constituents by Axisymmetric Surface Loading," Journal of Earth System Science, Vol. 118, No. 5, 2009, pp. 563-574.

[16] R. A. Schapery, "Approximate Methods of Transform Inversion for Viscoelastic Stress Analysis," Proceedings $4^{\text {th }}$ US National Congress on Applied Mechanics, Berkely, USA, 1962, pp. 1075-1085.

[17] C. W. Cryer, "A Comparison of the Three-Dimensional Consolidation Theories of Biot and Terzaghi," The Quarterly Journal of Mechanics and Applied Mathematics, Vol. 16, No. 4, 1963, pp. 401-412. 